\title{
On the Interpreter's Subjectivity in Business Interpreting from the Perspective of Skopos Theory-4.653
}

\author{
Mengqi Min $^{1} \&$ Feng Wang ${ }^{1}$ \\ ${ }^{1}$ School of Foreign Studies, Yangtze University, Jingzhou, China \\ Correspondence: Wang Feng, School of Foreign Studies, Yangtze University, No. 1, Nanhuan Road, Jingzhou \\ City 434023, Hubei Province, China. E-mail: wangfeng@yangtzeu.edu.cn
}

\author{
Received: March 2, $2018 \quad$ Accepted: March 9, $2018 \quad$ Online Published: April 19, 2018 \\ doi:10.5539/ass.v14n5p111 URL: https://doi.org/10.5539/ass.v14n5p111
}

\begin{abstract}
Business interpreting requires the interpreter to fully display his subjectivity to ensure the success of interpreting, besides traditional criteria of faithfulness and smoothness. However, the interpreter's subjectivity has not been fully recognized and studied. After a brief introduction to the two main concepts -- skopos theory and interpreter's subjectivity, three rules in skopos theory and three important interpreting strategies are discussed with the characteristics of business interpreting. Under the purpose-oriented skopos theory, the strategies mentioned in business interpreting are proved reasonable and effective, and the interpreter's subjectivity before, during and after interpreting plays a very important role in successful interpreting.
\end{abstract}

Keywords: interpreting; subjectivity; skopos theory

\section{Introduction}

With the development of globalization, the demand of intercultural communication has been growing over time, especially in China where the opening-up and reform have undergone unprecedented development and her status as the world's economic superpower is increasingly consolidated. Business interpreting, as a newly emerging profession linking all nations' economic activities together is playing a very important role in both domestic and international economic and trade activities. Business interpreters are working actively in almost all kinds of intercultural economic occasions such as business negotiation, exhibition, conference interpreting, foreign guests reception, and contract discussion.

However, though the interpreter is very important during the process of business interpreting, the interpreter's subjectivity has not been fully recognized and studied. Traditional interpreting studies mostly focus on strategies, and emphasize the interpreter's "loyalty" and "equivalence". They rarely discuss the interpreter's subjectivity. It seemed that the interpreter was just an "invisible interpreting mediator" (Valero-Garcés, 2007). Though Due (2015) proved that the interpreter is not an invisible being, however, it's from the perspective of its impact. With the rise of cultural turn in the 1970s, the interpreter's subjectivity has gained more and more attention from scholars both at home and abroad. Various ideas are challenging the traditional concept of translation and interpreting. This article aims to explore the interpreter's subjectivity in business settings from the perspective of skopos theory.

\section{Previous Researches on Skopos Theory}

\subsection{A Brief Introduction to Skopos Theory}

"Skopos" in Greek means "purpose". From the name skopos, we can see clearly that skopos theory emphasizes that all the translation activities and the strategies translators use during the process of translating should serve the goal of "purpose". Skopos theory was developed in the late 1970s in Germany and marked a general shift from predominantly linguistic and rather formal translation theories to a more functionally and socio-culturally oriented concept of translation (Baker, 1998:235). Hans Vermeer, under the guidance of Katharina Reiss, founded skopos theory, while other major advocators include Justa Holz-Mänttäri and Christiane Nord. Katharina Reiss, in Translation Criticism: The Potentials and Limitations: Categories and Criteria for Translation Quality Assessment, states that "text function" should be viewed as one of standards for translation criticism. She called the ideal translation mode as "integral communicative performance", which means the translated texts should be equal to the original texts in "content, language mode and communicative function" 
(Reiss, 1989:114). But as an experienced translator, she also admitted that equivalence is impossible under certain circumstances and not necessary. She believed that the communicative function of the target language is more important than the theory of Equivalence.

Hans Vermeer, a student of Katharina Reiss, abandoned the equivalence theory and further developed his teacher's theory. He believed that the purpose of translation is the most important element in translation actions. Translation means "to produce a text in a target setting for a target purpose and target addressees in target circumstances" (Vermeer, 1987:29). Skopos theory was first introduced in the Groundwork for a General Theory of Translation (1978) coauthored by Hans Vermeer and Katharina Reiss. It believed that translation is a communicative activity with certain aims. Based on skopos theory, Justa Holz-Mänttäri established the translation action theory which focuses on translation as a more complicated activity and the influences of roles like initiators, interpreters and readers. To her understanding, translation becomes a complex action designed to achieve a particular purpose. Kussmaul (1995) explained that: "the functional approach has a great affinity with Skopos theory. The function of a translation depends on the knowledge, expectations, values and norms of the target readers, who are again influenced by the situation they are in and by the culture. These factors determine whether the function of the source text or passages in the source text can be preserved or have to be modified or even changed." Christiane Nord (1997) perfected the functional translation theory by adding "faithful" to it. Under the guidance of "purpose decides everything", it seemed that the translators and interpreters can do anything as they want without any limitations. In this way, it would result in unfaithfulness between the original and target language. Therefore, she proposed faithfulness principle to skopos theory, which in return becomes more mature.

\subsection{The Three Rules of Skopos Theory}

Skopos theory has three main rules with different importance, which are the skopos rule, the coherence rule, and the fidelity rule.

\subsubsection{The Skopos Rule}

The Skopos rule takes the first place in skopos theory, and it can be summed up as "the end justifies the means" (Nord 1997: 124). Each translation is produced for a certain purpose and serves this purpose. Therefore, the translation should be able to function fully according to the purpose under certain circumstances and work well on the receivers it aims at. Directing at different types of texts with various text functions, the translator's translation methods and strategies differ. For example, advertising slogan translation should be easily spoken from everybody' lips. Legal documents translation requires accuracy. But for children's literature, the translation needs to be full of vividness and the language be as simple as possible.

\subsubsection{The Coherence Rule}

The coherence rule is also called intratextual coherence rule, it means the translation ought to be coherent and it requires the translator or interpreter to produce a text that can be understood by receivers under their specific background knowledge and environment (Nord 1997:32). This is, in translation practice, the translator or interpreter not only needs to fully understand the meaning of the source language, but also takes into consideration the target-receiver's cultural background, as well as the contents involved in the source language, then carry out the translation in accordance with the characteristics of the source language, rather than translate it stiffly into the target text.

Because interpreting has the feature of face-to-face communication, the interpreter should observe closely the speaker's body movement, facial expression, tone of the voice, etc. These nonverbal communication symbols reflect certain cultural attributes, and the interpreter can present these figures through interpreting strategies. For example, the gesture of crossing the middle finger and index finger means praying for good luck for Americans. The interpreter can explain the meaning of this gesture to the audience. Again, Japanese see eye-contacting as a challenge to others, so the interpreter should remind the counterparts while talking with Japanese (Onken, 2018).

\subsubsection{The Fidelity Rule}

In the fidelity rule, the source text and target text should be in intertextual coherence (Nord, 1997:32). The fidelity rule requires equivalence on the function of source and target text, and be as faithful as possible. But the degree of fidelity is decided by the purpose and how the translator or interpreter understands the source text. In business interpreting, the interpreter's subjectivity is restricted by the rule of fidelity. The interpreter cannot interpret casually, carelessly or distort the intention of the speaker.

To conclude, the skopos rule, coherence rule and fidelity rule are all fundamental rules of skopos theory, however, they are of different importance. According to Nord, the coherence rule and fidelity rule are 
subordinate to the skopos rule, and the fidelity rule is subordinate to the coherence rule (Nord, 1997:24). Under normal conditions, the skopos rule and coherence rule are consistent, but there are always conflicts between the fidelity rule and the former two rules. When this happens, what the translator or interpreter ought to do is to sacrifice the fidelity rule and preserve the skopos rule and the coherence rule.

\section{The Interpreter's Subjectivity}

\subsection{Definitions of Subjectivity}

Generally speaking, the study of the interpreter's subjectivity in China is influenced by western studies. In Chinese translation studies, the importance of the interpreter's subjectivity has not been fully recognized. Early studies about subjectivity believed that the subjects of translation are the author, the translator and the reader. But nowadays, mostly the translator and the reader are considered the subjects of translation (Zha \& Tian, 2003:21). For subjectivity, there's no consistent explanation. However, the following five definitions can be seen as references: subjectivity is human nature; subjectivity is equivalent to subjectiveness; the essential character of subjectivity is its practice quality; subjectivity refers to a kind of relationship in the practical activities of subjects; subjectivity means possession of self-determination, dynamic role and creative power, or in other words it is a synthesis of independent self-determination and subjective dynamic role (Feng, 1994:45-61). All these five definitions can be seen as understandings of the essence of subjectivity, and the last one is often regarded as the most scientific. More specifically, subjectivity is the externalization of its essence and it plays an active role in remolding, influencing and controlling the object and making the object serve the subject (Wang, 1995:35).

As one of the subjects of interpreting, the interpreter has his personal subjectivity that can be seen from two sides: for one thing, the interpreter is a living entity capable of thinking and feeling. He has consciousness and self-esteem and when conflicts come from external, these characteristics become obvious. The inner subjectivity of the interpreter rests with external influences. Thus, subjectivity in this article pays equal attention to the inner mechanism as well as the inner response to external impetus. Moreover, the interpreter's subjectivity, on one hand, consists of inner elements such as incentive, pride and attitude, etc., which are rooted in his inner ego. On the other hand, it is based on his response to the external factors which are a collection of all kinds of influences. To put it in another way, the interpreter's subjectivity is not only inner and personal, but social and cultural.

\subsection{The Exertion of the Interpreter's Subjectivity}

Interpreting is different from translation, because it is a face-to-face and active communication. Both parties are present to the interpreter, so the information can be transferred quickly and frequently to accomplish a successful and fluent conversation.

\subsubsection{Subjectivity Before Interpreting}

Any form of interpreting needs good preparation, and business interpreting is no exception. In the process of such preparation, the interpreter must begin to exert his "subjectivity". Upon receiving the interpreting task, the interpreter should start preparing in time to ensure that the task can be accomplished successfully. Compared to the interpreting time, this process of preparation can last quite longer, during which the interpreter's subjectivity has already been unlocked. If the interpreter can exert his subjectivity actively, flexibly and suitably, he can undertake the desired interpreting effectively. About the specific works needed for preparation, "before the interpreting started, the interpreter should be fully prepared, including being familiar with the interpreting site and equipments, the interpreting themes, scripts and relative terms, as well as the clients, physical and psychological preparation, among which the full understanding and preparation about the themes and clients are of great importance" (Bao, 2012:121-123). The interpreter should be carefully prepared for the interpreting themes in advance. These contents show that the interpreter's subjectivity is performed from different aspects and angles, and at the same time the subjectivity's exertion is not a simple or once-for-all process, but a dynamic process containing the involvements of many factors. It requires the interpreter's dedication to the interpreting and the habits formed in former interpreting training. Subjectivity before interpreting can be divided into four kinds as follow:

\section{1) Collecting Background Information}

After receiving the interpreting work, the interpreter's job is to figure out what the theme is, who the speakers are and where they are from. By doing so, the interpreter can accumulate extra knowledge for later interpreting as well as form a broad understanding of what accents the speakers may have. In addition, if the speakers have scripts, the interpreter can ask them to provide them. For instance, if the theme of the conference is clean energy, the interpreter should refer to books or online resources to learn about the special terms and background knowledge about clean energy. To be more specific, the interpreter shall know his clients, including their 
personal information. The name, gender and position are basic things he needs to know. An excellent interpreter will make the best use of subjectivity and try to use all the resources he can find to search for the clients' personal hobbies, educational and cultural backgrounds, basic family information, his company's culture and relevant information about his position and so on. The reason why the interpreter should do this is that business interpreting is direct face-to-face communication with customers, including consecutive interpreting and simultaneous interpreting.

\section{2) Knowledge Accumulation}

To be equipped with a large vocabulary is a difficult process and sometimes brings a big challenge to the interpreter. Only when the interpreter uses his subjectivity can he make this task easier. The interpreter is commonly called Jack of all Trades, which means that he has broad knowledge in many fields, although not quite professional. Generally speaking, popular topics like environmental protection and daily liaison are easier to manage, but if the subjects are Chemistry, Mechanics, business negotiation and other professional fields, the difficulty of interpreting will increase greatly because most of the interpreters are from literal arts. In this case, the only thing that the interpreter can do is to exert his subjectivity and try to accumulate professional vocabulary and knowledge in this field.

\section{3) Familiarity with the Interpreting Site}

To be familiar with the interpreting site in advance is very important. If the interpreting is quite formal, and if situation permits, the interpreter should be at the interpreting site at least one day in advance and keep in touch with persons in charge, to ensure the interpreting will have a good effect. Most of the interpreting take place at grand hotels and big international conference centers. Thus, if the interpreter is not familiar with the workplace, this will result in being late caused by traffic problems, or influence the interpreter's mental status. Moreover, considering the excitements the interpreter may have for meeting some celebrities, the results can be worse. It can even change the result of the business conference. If for a variety reasons the interpreter is not able to check the working environment personally, he can seek help from internet or teachers and friends. In a word, no matter by which approach, the interpreter must be familiar with the traffic and other things for the interpreting.

\section{4) Etiquette Learning}

During the process of learning interpreting etiquette, the interpreter can exert subjectivity properly. In the face of customers from different countries and different cultures, the interpreter also needs to study the unique cultural etiquette in its culture, as well as the necessary and basic etiquette. "The so-called mastering a foreign language not only means mastering its grammar and vocabulary but also means understanding the cultural background, the elegance, vulgarity, humor, exaggeration and rhetorical characteristics of the language, etc.” (Zhang, 1999)

China is renowned for valuing ritual and ceremonies ever since ancient times. For Chinese people, etiquette can reflect a person's education and taste. It is necessary for the interpreter who always meet foreign guests and political celebrities to learn etiquettes. When interpreting for foreign guests, the interpreter should act naturally and gracefully, neither arrogant nor servile. While facing political celebrities, the interpreter should act calmly and smartly, showing them the knowledge and etiquette gained through hard training. The learning of etiquette itself is an art which contains the way of sitting and walking, hand movement, facial expression, personal image, appearance, cloth, social etiquette, dining etiquette, wine-tasting, and so on. The first thing for the interpreter to pay attention to is appearance. For instance, men's suits cover a lot of details that deserve carefully consideration. Different occasions have different standards for suits' buttons, ties' color, color match of pants and socks, accessories, and so on. Therefore, the interpreter needs to take every aspect into account, and uses them effectively.

\subsubsection{Subjectivity during Interpreting}

The interpreter's subjectivity is displayed most remarkably in the process of interpreting. According to different types of interpreting, the interpreter ought to adjust his specialized vocabulary, communication style, dressing, etc. Before interpreting, the interpreter needs to put himself in the speaker's position, and to think carefully about what topics or questions may arouse his interests, and while interpreting, combine the theme with the purpose of activity to help the client solve the problems. For instance, if the client is a professor or an officer, the interpreter should avoid using over commercialized information when talking with them. In addition, the first meeting with foreign clients is of vital importance, because it doesn't involve difficult and deep technical problems. Thus, it can be seen as a preheat before the start of the interpreting as well as a good opportunity for clients to know the interpreter.

Displaying the interpreter's subjectivity has a pivotal role on interpreting practice. There exist different cultural 
thinking modes and idiomatic expressions between different languages and habits. While the interpreter is decoding and transforming two languages, he is also using his interpreting strategies and methods to reach good communication effects. Moreover, while interpreting, as the time is short, the interpreter is under high pressure and has to fulfill high requirements; he has no time for careful consideration or long hesitation. Because of this, how the interpreter uses his subjectivity to achieve the goal is very important.

As is known to all, many business negotiations are time-consuming and often become long-drawn-out fights. When an agreement cannot be reached, the parties would probably go through some changes in their mood and even would lose control and say something impolite. Therefore, the interpreter as the bridge linking both parties cannot just interpret the original language simply and directly. For example,

The Chinese sentence “你们一点诚意都没有, 我们只能这个价, 否则就别谈了” can be translated into: 1) “You have no sincerity, this is the prize we can give. If you don't accept, we will end this negotiation." 2) "If this is the case, we would say it is unwise of you to refuse this price." Both translations are right, but in different situations, the translations will be inappropriate. If the speaker has made his mind after long-time negotiation and he cannot see the possibility of success, the first translation can correctly express his meaning. However, if the speaker intends to use these words as a treat, then the second translation is better. All these translations can find its theoretical foundation in skopos theory. Skopos theory provides to translation critics another approach rather than judging the translation by a single standard. In this way, whether the interpreting function works or not determines the success or failure of the interpreting. To achieve the goal of interpreting, the interpreter should adjust the interpreting criteria according to different texts, readers, different requirements from the clients, etc.

\subsubsection{Subjectivity after Interpreting}

For the interpreter, reviewing is always an important part of interpreting that can not be ignored. Only by reviewing the interpreting task repeatedly can the mistakes be reduced to the minimum in the future. This process can be time-consuming and tedious; however, the interpreter needs to pay enough attention to it. "Reviewing after interpreting" shows the interpreter's final subjectivity in interpreting. At the same time, it helps the interpreter to form his own personalized interpreting styles.

\section{Characteristics of Business Interpreting}

Among all those forms of interpreting, every type has its own characteristics that are different from other kinds. Business interpreting is a new form of interpreting that developed with economic globalization. It shares many similarities with common interpreting, but more importantly, it has its own characteristics.

\subsection{Specialization}

The language used in business interpreting requires high specialization. The language forms, words, contents are closely related to business. The language form is not changeable and the meaning of words are jargons sometimes. In addition, business English stresses simplicity, formality and internationality on the use of words, and rejects abstract or ambiguous expressions, as shown in the expression, "Payment by irrevocable L/C is convenient for us." The irrevocable $\mathrm{L} / \mathrm{C}$ is a form of payment; it means any changes (amendment) or cancellation of the LC (except it is expired) is done by the applicant through the issuing bank. It must be authenticated and approved by the beneficiary. If the interpreter doesn't have the basic knowledge in foreign trade terms, it would be impossible for him to make both parties understand. Take another example, "short delivery" is a glossary in international business trade; it means that the quantity received is less than the quantity listed. Without the abundant accumulation of foreign trade knowledge, the interpreter is very likely to interpreter it as "quick delivery". In this regard, the first principle of business interpreting should be "accurate", which means he cannot break away from the speaker's purpose. Considering that there are lots of business terms and articles of laws in business interpreting, the interpreter needs to try his best to make the interpretation concise and formal and make sure the words used are normative and professional.

\subsection{Efficiency}

In international business activities, especially in business negotiations, obtaining economic benefits is the priority, and gaining more profit and value is the core of negotiation. The competition and profit-orientation of business make all the parties stick to their own positions and try to persuade others. When the speaker's way of expression is not adept or he is not paying enough attention to the emotional factors, the interpreter should adapt the policy of intervention and act as a lubricant to make the negotiation procedures efficient and effective. To achieve efficiency, sometimes it would be unnecessary to repeat redundant information. However, what information is redundant and what is not are not easy to judge. It needs the interpreter's subjectivity to make a decision and help the parties involved to conclude business negotiations effectively. 


\section{Interpreting Strategies Regarding Subjectivity}

According to Gile (1995), C = KL+ELK, where C, KL and ELK stand for comprehension, linguistic knowledge and extra-linguistic knowledge respectively. In other words, comprehension of the source information is based on linguistic and extra-linguistic knowledge. It indicates that for any form of interpreting, the first and foremost thing is comprehension, based on which the interpreter adjusts the interpreting contents by displaying the interpreter's subjectivity. That is, after fully comprehending the clients' interpreting contents, the interpreter's work is to analyze the source language by using his knowledge for the language and extra-linguistic knowledge, and this is where the origin of subjectivity lies. Furthermore, there are three most important specific methods for the display of the interpreter's subjectivity.

\subsection{Addition}

Addition is a frequently used technique in translation as well as in interpreting, but because interpreting is once-off and instant, this approach should not be used too often in case it would increase the interpreter's burden so much that other important messages in interpreting are lost. There are two situations for using addition. First, when different cultural backgrounds cause barriers for mutual understanding, addition can help make extra explanation. For example, Americans loves dogs, thus, in their culture, a dog represents bravery and loyalty. There are many phrases and slangs containing the word "dog". An American would probably call a male friend who is a little bit fat "big dog". In this situation, if the listener doesn't know this cultural background, the interpreter should give extra explanation about the dog's special image in American culture. From this example, we can also see how the interpreter applies the coherence rule of skopos theory.

Second, when different language types have differences on language terms, addition can make extra explanation. According to theories of language morphology, all the languages can be divided into analytic and synthetic language. Chinese belongs to the former, while modern English the latter. Moreover, English emphasizes hypostasis, frequent conjunctions, and more noticeable logical connectives, while Chinese emphasizes parataxis and much fewer conjunctions. Consequently, especially in Chinese-English interpreting, addition is often used.

\subsection{Omission}

To a large extent, omission is used in business interpreting to make the conversation smooth and efficient. The interpreter would leave out some useless information or content to make the target receiver better understand more important messages. In business interpreting, some less important adjectives, adverbs can be omitted, but those important and material contents such as names, positions, names of organization, time, place, number, etc., shall be interpreted precisely. Experienced interpreters would unconsciously omit some empty words like "well", "so", "I think", etc.

\subsection{Reorganization}

Reorganization means the readjustment and integration of the interpretation. It reduced the differences caused by habitual ways of expression in intercultural communication and lowered the difficulties resulted from different understanding in the thinking modes. In this process, the interpreter should display subjectivity fully and actively. Reorganization is used most frequently and typically in science and technology-related topics. When the interpreter has received a work of science, except remembering necessary special terms, he needs to reorganize long and difficult sentences or complicated sentence patterns. This requires the interpreter to be equipped with a solid ability of language analysis as well as a good master of sentence patterns and linguistic characteristics.

\section{Conclusion}

Nowadays, with unprecedented intercultural communication across the world, people all over the world are paying increasing attention to the frequent exchanges in politics, economy, and culture. Interpreting is playing a very important role in bridging the language gap, assisting people from different language communities and cultural backgrounds to communicate efficiently with each other and make the "global village" firmer and bigger. Business interpreting is a very important filed in recent research. After a brief introduction to the two main concepts -- skopos theory and the interpreter's subjectivity, three rules in skopos theory and three important interpreting strategies are discussed with the characteristics of business interpreting. Under the purpose-oriented skopos theory, the strategies in business interpreting are proved reasonable and effective, and the interpreter's subjectivity before, during and after interpreting is very important in successful interpreting. The limitation of this article is that examples are not enough and the combination of skopos theory with the interpreter's subjectivity still needs further research. 


\section{References}

Baker, M., \& Malmjaer, K. (1998). Routledge Encyclopedia of Translation Studies. London and New York: Routledge.

Bao, L. (2012). The reconstruction of cultural presupposition in liaison interpreting. Journal of Hubei University of Economics (Humanities and Social Sciences), (5), 121-123.

Due, C., \& Gartley, T. (2015). The interpreter is not an invisible being: a thematic analysis of the impact of interpreters in mental health service provision with refugee clients. Australian Psychologist. https://doi.org/10.1111/ap.12181

Feng, Z. Y. et al. (1994). A Theory on Subjectivity: A New Anthropology of a New System in a New Era. Beijing: Peking University Press.

Gile, D. (1995). Basic Concepts and Models for Interpreter and Translator Training. Amsterdam: John Benjamins Publishing Company.

Kussmaul, P. (1995). Training the Translator, John Benjamins Publishing Co.

Nord, C. (1997). Translating as a Purposeful Activity. Manchester: St. Jerome Publishing.

Onken, M. (2018). Business in Japan: Non-verbal Communication. Retrieved February 28, 2018, from http://www.onken.com/classroom/internationalmanagement/Japan/Nonverbalcommunication.html

Reiss, K. (Translated by Erroll. F. Rhodes.) (1989). Translation Criticism: The Potentials and Limitations. Manchester: St. Jerome, Publishing.

Valero-Garcés, C. (2007). Challenges in multilingual societies. the myth of the invisible interpreter and translator. Across Languages \& Cultures, 8(1), 81-101. https://doi.org/10.1556/Acr.8.2007.1.5

Vermeer, H. J. (1987). What does it mean to translate? Indian Journal of Applied Linguistics, 13(2), 25-33.

Wang, Y. L. (1995). On the basic connotation and characteristics of subjectivity. Tianfu New Idea, 6, 34-38.

Zha, M. J., \& Tian, Y. (2003). On the subjectivity of the translator. Chinese Translators Journal, (1), 19-24.

Zhang, W. W. (1999). English-Chinese Simultaneous Interpreting. China Translation Corporation.

\section{Copyrights}

Copyright for this article is retained by the author(s), with first publication rights granted to the journal.

This is an open-access article distributed under the terms and conditions of the Creative Commons Attribution license (http://creativecommons.org/licenses/by/4.0/). 